Article

\title{
Expression of Elongation Factor (EF)-Tu Is Correlated with Prognosis of Gastric Adenocarcinomas
}

\author{
Chaoyang Xu ${ }^{1,2, *}$, Jianjun Wang ${ }^{3}$, Jiajia $\mathrm{Li}^{4}$ and Rengui Fang ${ }^{3}$
}

1 Department of Breast and Thyroid Surgery, Shaoxing People's Hospital, Shaoxing Hospital of Zhejiang University, Shaoxing, Zhejiang, 312000, China

2 Department of Surgical Oncology, Sir Run Run Shaw Hospital, College of Medicine, Zhejiang University, Hangzhou, Zhejiang, 310016, China

3 Department of Surgical Oncology, The Chunan County First Hospital, Chunan, Zhejiang, 311700, China; E-Mails: wjj0714@163.com (J.W.); fang_rengui@yahoo.com.cn (R.F.)

4 Department of Surgery, Shaoxing People's Hospital, Shaoxing Hospital of Zhejiang University, Shaoxing, Zhejiang, 312000, China; E-Mail: dr_xuchaoyang@yahoo.com.cn (J.L.)

* Author to whom correspondence should be addressed; E-Mail: xuchaoyang2001@ @126.com; Tel.: +86-571-86006912; Fax: +86-571-86044817.

Received: 10 August 2011; in revised form: 16 September 2011 / Accepted: 22 September 2011 / Published: 10 October 2011

\begin{abstract}
Altered expressions of mitochondria elongation factor $\mathrm{Tu}(\mathrm{EF}-\mathrm{Tu}$ ) have been observed in certain types of cancers, including gastric cancer cell lines, but the impact of the alterations in gastric adenocarcinoma remains unclear. The purpose of this study was to investigate the expression of EF-Tu in gastric adenocarcinoma and to assess its clinical significance. A total of 104 paired resected gastric adenocarcinoma and corresponding normal specimens were collected in this study. EF-Tu expression was assessed by immunohistochemical staining. The correlation of EF-Tu expression and patients' clinicopathological parameters was statically evaluated and the prognostic significance of EF-Tu expression was assessed by univariate and multivariate analyses. Forty-nine out of $104(47.1 \%)$ gastric adenocarcinoma specimens showed high expression of EF-Tu, while the remaining 55 specimens showed weak or negative expression of EF-Tu. In contrast, EF-Tu high expression was detected in 62.5\% (65 of 104) normal tissues. Down-regulation of EF-Tu was associated with serosal invasion $(P=0.042)$ and node involvement $(P=0.005)$, and down-regulation of EF-Tu was correlated with poor overall survival $(P=0.020)$. In curative resection $(\mathrm{R} 0)$ patients, there were also significant differences
\end{abstract}


$(P=0.043)$. In the multivariate analysis, the EF-Tu expression remained a significant independent prognostic factor $(P=0.038)$. Our results indicate that EF-Tu is expressed in both gastric adenocarcinoma and corresponding normal tissues. Down-regulation of EF-Tu expression is associated with advanced disease stage and EF-Tu expression maybe served as an independent prognostic factor.

Keywords: elongation factor Tu; gastric cancer; survival

\section{Introduction}

Currently gastric adenocarcinoma remains one of the most common cancers worldwide, and one of the leading causes of cancer-related death in China [1]. Gastric adenocarcinoma encompasses many subtypes with distinct genetic and biological features. Identification of new biological markers to determine the risk of poor prognosis is important for designing treatment strategies $[2,3]$.

Elongation factor $\mathrm{Tu}$ (EF-Tu, Tu translation elongation factor, TUFM), one of the most abundant proteins of the mitochondrial, plays a key role in the elongation process of mitochondrial protein biosynthesis [4]. The main function of the translation factor EF-Tu is to deliver aminoacyl-tRNA to the A-site on the ribosome. In addition, other functions and characteristics have been reported for EF-Tu, including its chaperone properties [5,6] and roles in signal transduction [7]. Expressions of EF-Tu have been observed in a variety of tissues with high level of expression in tissues with intrinsically active oxidative metabolism such as heart [8] and brain [9].

Recent studies have shown that the expression level of EF-Tu is altered in several types of cancers. Up-regulation of both EF-Tu and the cytoplasmic elongation factor EF-1a in human pancreatic adenocarcinoma has been reported [10,11], whereas absence of EF-Tu expression in human hepatocellular carcinoma cell lines HepG2 was also observed [12]. These studies yielded variable and, in some cases, contradictive results in terms of the expression of EF-Tu in different tumor cells [11-13]. Furthermore, one recent study showed that EF-Tu was up-regulated in human gastric cancer cell line AGS compared to the rat normal gastric cell line RGM-1, and it was then proposed that EF-Tu might be served as potential cancer biomarkers in mitochondria [14].

The purpose of the present study was to examine the expression status of EF-Tu in gastric adenocarcinoma tissues and to evaluate whether EF-Tu expression level correlates with the clinicopathological parameters and prognosis of gastric adenocarcinoma patients. To the best of our knowledge, this is the first report on evaluating expression of the EF-Tu in clinical specimens of gastric adenocarcinoma. Our results indicate that EF-Tu down-regulation correlates well with advanced disease stage and EF-Tu expression is an independent prognostic factor.

\section{Materials and Methods}

\subsection{Case Selection}

Paired specimens of gastric adenocarcinoma and corresponding normal specimens were obtained from 104 patients who underwent surgical resection of gastric adenocarcinoma in the department of 
surgical oncology, Sir Run Run Shaw Hospital, Zhejiang University College of Medicine between July 1995 and March 2007, with informed consent under the guideline of Hospital Ethics Committee. Among the 104 patients, 13 patients who had synchronous peritoneal metastasis were included in this study. The patients comprised 68 males and 36 females aged 38 to 88 years old (mean $=67.5$ years old). None of the patients received any anti-cancer treatment before surgery. Correlation between expression of EF-Tu and clinicopathologic parameters including age, sex, pTNM pathological classification of the International Union against Cancer (UICC) [12], peritoneal dissemination, and lymph node metastasis were evaluated.

\subsection{Immunohistochemistry}

Immunohistochemical analysis for EF-Tu expression was performed on formalin-fixed, paraffin-embedded sections of surgical specimen. The slides were deparaffinized in xylene and rehydrated in gradient ethanol solutions. Endogenous peroxidase was blocked with $0.3 \% \mathrm{H}_{2} \mathrm{O}_{2}$ in methanol for $10 \mathrm{~min}$. The slides were immersed in $10 \mathrm{mM}$ citric buffer $(\mathrm{pH} \mathrm{6.0)}$ with heating for 15 min for antigen retrieval. The slides were cooled at room temperature for $20 \mathrm{~min}$ and then washed with phosphate buffered saline (PBS). Nonspecific binding was blocked by preincubation with $10 \%$ fetal calf serum in PBS with $0.01 \%$ sodium azide, and the slides were incubated in a humid chamber for $1 \mathrm{~h}$ with antibody against EF-Tu (mouse monoclonal, CBP-KK1, Santa Cruz; 1:200). After washing three times in PBS, the slides were incubated with the EnVision-HRP complex (undiluted, DAKO) for $60 \mathrm{~min}$. The slides were visualized with diaminobenzidine (DAKO Corp., Changzhou, China) and then counterstained with hematoxylin. For substitute negative controls, the primary antibody was replaced with phosphate buffered saline. Positive controls included gastric adenocarcinomas tissue known to exhibit high levels of marker.

All the slides were examined and scored independently by two experienced pathologists to avoid subjective biases. The criteria for EF-Tu expression level was as follows: no cytoplasm staining was given a score 0 ; faint/barely perceptible cytoplasm staining detected in $>25 \%$ of tumor cells was scored as 1+; a moderate cytoplasmic staining observed in $>25 \%$ of tumor cells was scored as $2+$, a high cytoplasmic staining observed in $>25 \%$ of tumor cells was graded $3+$ respectively. A score of 0 was considered negative and $1+$ was considered weak positive, whereas $2+$ and $3+$ were considered high positive.

\subsection{Follow $U p$}

The patients were followed up until death or until the date of last follow-up of 30 November 2007. Thirty-six patients $(34.6 \%)$ died during the follow-up period, and the median follow-up interval was 50.6 months (range: 3 to 127 months).

\subsection{Statistical Analysis}

All statistical analyses were conducted using the statistical program SPSS 15.0 for windows (SPSS, Chicago, IL, USA). Pretreatment characteristics were analyzed using the 2-tailed chi-square test, and the 2-tailed t test was used to evaluate correlation between EF-Tu expression and clinicopathologic 
parameters. Univariate analysis of patient survival was performed using Kaplan-Meier method. The survival curves were compared using the log-rank test. Multivariate analyses were done using logistic regression and Cox's proportional hazard model. The accepted level of significance was set as $P<0.05$.

\section{Results}

\subsection{EF-Tu Expression Correlates with Clinicopathologic Parameters}

The majority (94.2\%, 98 of 104) of gastric adenocarcinomas showed positive expression of EF-Tu. In a total of 104 gastric adenocarcinoma specimens, 55 (52.9\%) showed high EF-Tu expression, $39(41.3 \%)$ showed weak EF-Tu expression and 6 (5.8\%) showed absence of EF-Tu expression (Figure 1). EF-Tu was also observed in 103 (99\%) corresponding normal tissues with 65 (62.5\%) showed high expression and 38 (36.5\%) showed low expression. Lymphocytes, vascular endothelial cells, and smooth muscle also showed high EF-Tu expression (Figure 2).

EF-Tu expression was correlated with depth of invasion, and node involvement. However, no difference was found between both groups regarding sex, age, tumor differentiation, location, type of operation, ECOG, tumor size, distant metastasis (Tables 1 and 2).

Figure 1. Immunohistochemical analysis of elongation factor $\mathrm{Tu}(\mathrm{EF}-\mathrm{Tu})$ expression in gastric adenocarcinomas. (a) High complete cytoplasmic staining observed in $>25 \%$ of tumor cells; (b) faint/barely perceptible cytoplasmic staining detected in $>25 \%$ of tumor cells; (c) No staining observed.

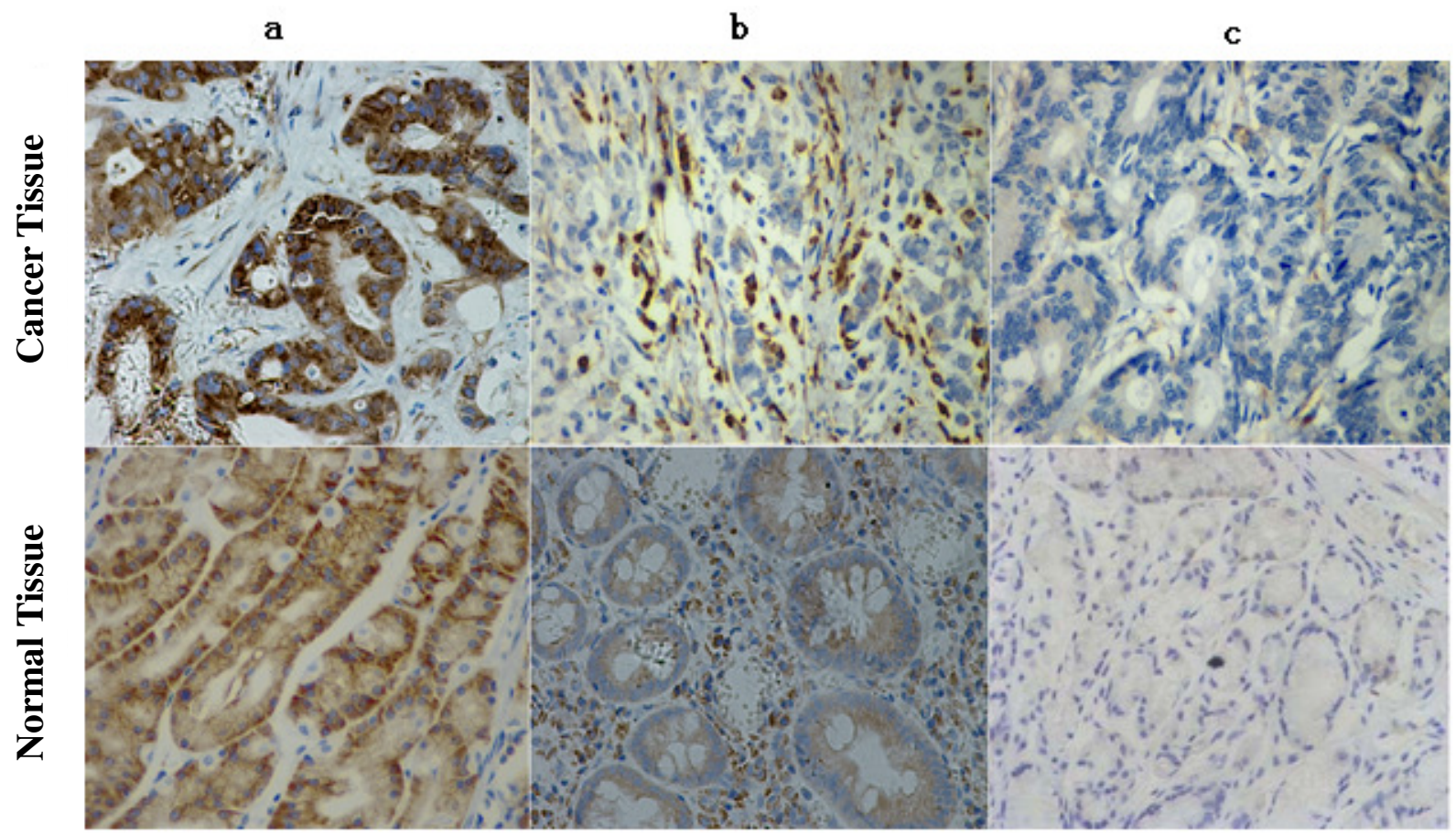


Figure 2. Immunohistochemical analysis of EF-Tu expression in interstitial tissue: (a) Smooth muscle; (b) venous endothelial cells; (c) artery endothelial cells, showed high EF-Tu expression. Immunohistochemical analysis of EF-Tu negative control: (d) Smooth muscle; (e) venous endothelial cells; (f) artery endothelial cells.

(a)

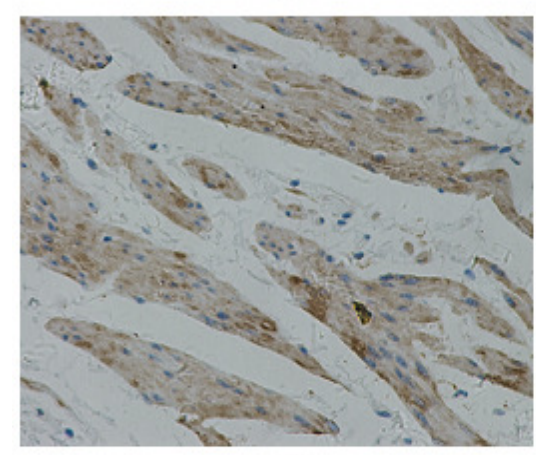

(d) (b)

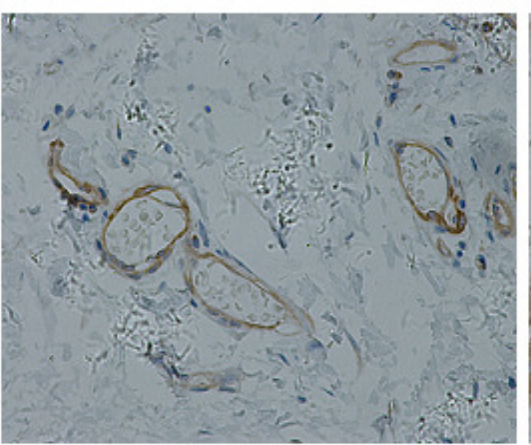

(e)

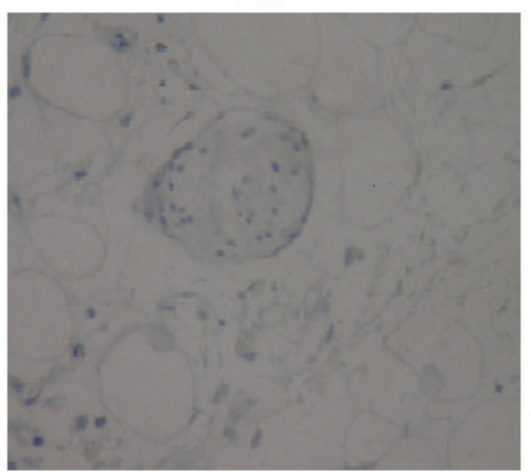

(c)

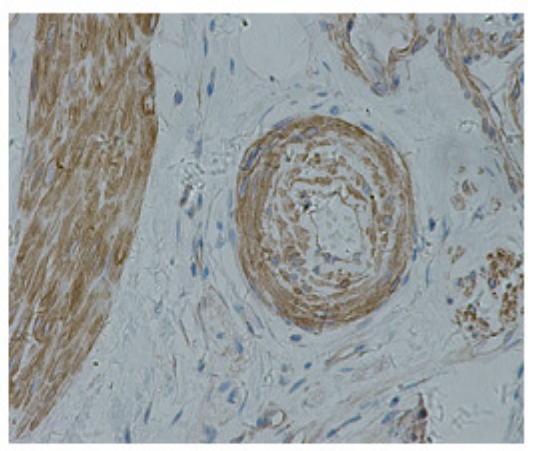

(f)

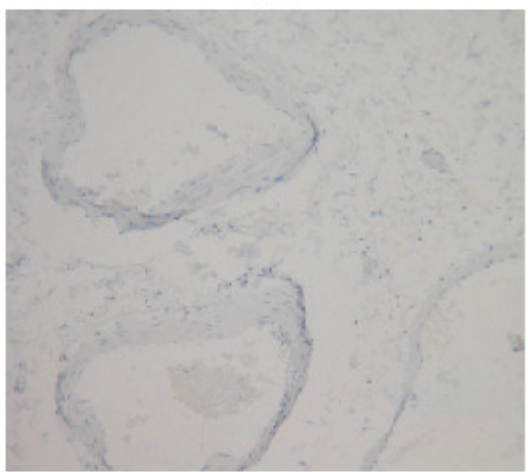

Table 1. Comparison of demographic and perioperative data in gastric adenocarcinoma patients.

\begin{tabular}{lccc}
\hline & Negative/low $(\boldsymbol{n}=\mathbf{5 5})$ & High $(\boldsymbol{n}=\mathbf{4 9})$ & $\boldsymbol{P}$ value \\
\hline Age & $54.9 \pm 11.3$ & $57.2 \pm 12.5$ & 0.329 \\
Gender & & & 0.104 \\
$\quad$ Male & $40(61.5 \%)$ & $28(51.7)$ & \\
$\quad$ Female & $15(38.5 \%)$ & $21(48.3)$ & \\
ECOG & & & 0.544 \\
0 & $18(32.7)$ & $17(35.4)$ & \\
1 & $37(67.3)$ & $31(62.5)$ & \\
2 & 0 & $1(2.1)$ & \\
Type of operation & & & \\
$\quad$ Total gastrectomy & $6(10.9)$ & $5(10.2)$ & \\
$\quad$ Subtotal gastrectomy & $49(89.1)$ & $44(89.8)$ & \\
\hline
\end{tabular}

ECOG: Eastern Cooperative Oncology Group; age represents as mean $\pm \mathrm{SD}$; values in the parenthesis are percentage. 
Table 2. Comparison of clinicopathologic features in gastric adenocarcinoma patients.

\begin{tabular}{lccc}
\hline & Negative/low $(\boldsymbol{n}=\mathbf{5 5})$ & High $(\boldsymbol{n}=\mathbf{4 9})$ & $\boldsymbol{P}$ value \\
\hline Location & & & 0.753 \\
$\quad$ Upper body or whole & $13(23.6)$ & $9(18.5)$ & \\
$\quad$ Lower or middle body & $42(76.4)$ & $40(81.5)$ & 0.061 \\
Tumor differentiated & & & \\
$\quad$ Well & $7(12.7)$ & $2(4.1)$ & \\
$\quad$ Moderate & $14(25.5)$ & $22(40.0)$ & \\
$\quad$ Poor & $34(61.8)$ & $25(55.9)$ & 0.021 \\
$\quad$ Tumor size & $5.3 \pm 2.3$ & $4.3 \pm 2.0$ & 0.042 \\
Serosal invasion & $14(25.5)$ & $22(44.9)$ & \\
$\quad$ No & $41(74.5)$ & $27(55.1)$ & 0.005 \\
$\quad$ Yes & & & \\
Node involveme & $13(23.6)$ & $25(51.0)$ & \\
$\quad$ No & $42(76.4)$ & $24(49.0)$ & 1.000 \\
$\quad$ Yes & 21 & 19 & \\
Mean no. of tLNs & & & \\
Distant metastasis & $48(87.3)$ & $43(23.6)$ & \\
$\quad$ No & $7(12.7)$ & $6(23.6)$ & \\
$\quad$ Yes & & $12(24.5)$ & \\
Normal specimens EF-Tu expression & $27(49.1)$ & $37(75.5)$ & \\
$\quad$ Negative/low & $28(50.9)$ & & \\
High & & & \\
\hline
\end{tabular}

tLNs indicates total retrieved Lymph Nodes; LN: Lymph Node.

\subsection{Univariate Analysis}

In univariate analysis tumor location, tumor size, node invasion, distant metastasis, type of operation and EF-Tu expression were associated with overall survival. Based on the expression level of EF-Tu, the mean overall survival time for negative/low EF-Tu expression group were 71.6 months and for high EF-Tu expression group, 101.7 months, respectively. The difference in survival was significant $(P=0.020)$ (Figure 3a). Even in the curative resection cases, a mean overall survival time for negative/weak EF-Tu expression group were 79.0 months, and for high EF-Tu expression group 106.2 months respectively. The difference in survival was also significant $(P=0.043)$ (Figure $3 b$ ). These data suggest down-regulated EF-Tu expression correlates with poor prognosis.

\subsection{Multivariate Analysis}

The following significant parameters were entered into a multivariate analysis: EF-Tu, distant metastasis, serosal invasion, number of mLNs, number of tLNs, type of operation, age, tumor size, histological type, gender, tumor location. In terms of both overall and recurrence-free survivals, the EF-Tu expression was the independent predictor (hazard ratio $0.460, P=0.038$ Table 3), which suggests EF-Tu expression served as an independent prognostic factor. 
Figure 3. (a) The mean overall survival time for the negative/weak EF-Tu expression group was 71.6 months, and for the high EF-Tu expression group was 101.7 months $(P=0.020)$; (b) In R0 (radical operation) gastric cancer, the mean overall survival time for the negative/weak EF-Tu expression group was 79.0 months and for the high EF-Tu expression group was106.2 months $(P=0.043)$.
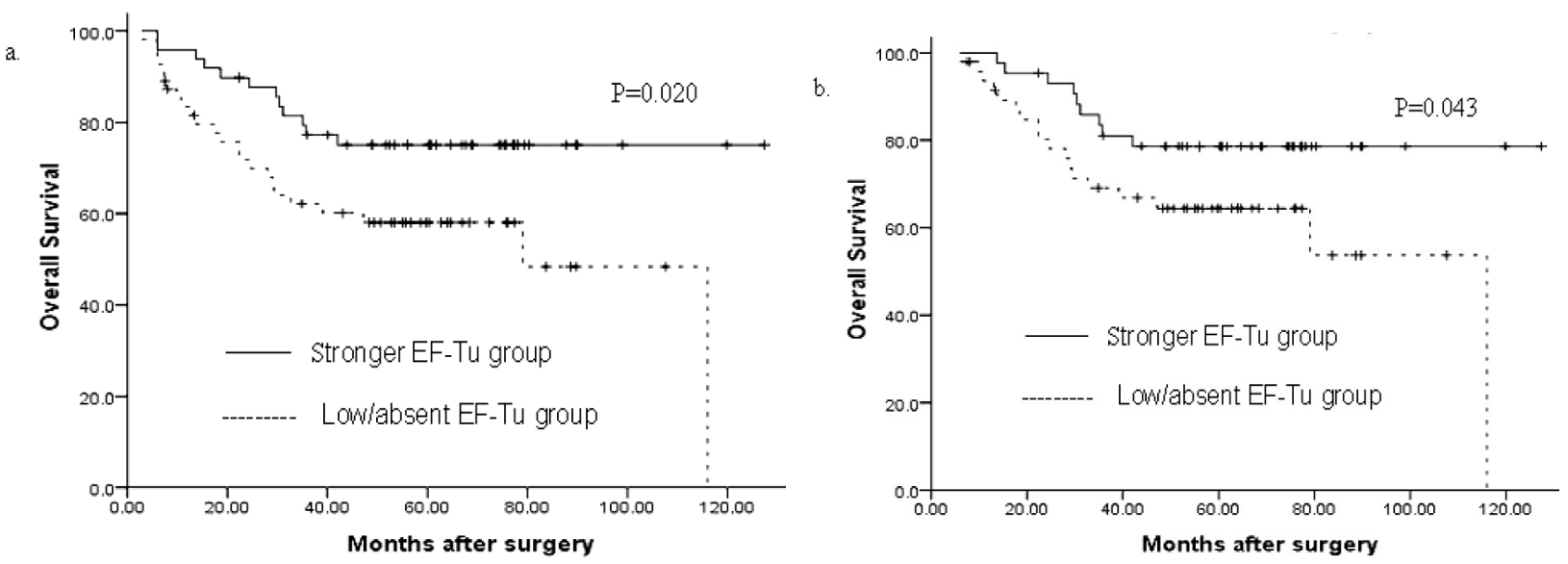

Table 3. Results of Stepwise multivariate analysis for Prognostic Factors $(n=104)$. Values in parentheses are $95 \%$ confidence intervals. * Cox proportional hazards model.

\begin{tabular}{lccc}
\hline \multicolumn{1}{c}{ Variable } & HR & $\mathbf{9 5 \%}$ CI & $\boldsymbol{P} *$ value \\
\hline EF-Tu & 0.460 & $0.220-0.960$ & 0.038 \\
Distant metastasis & 4.323 & $1.442-12.963$ & 0.009 \\
Serosal invasion (yes $v s$. no) & 6.242 & $1.808-21.548$ & 0.004 \\
mLNs & 1.082 & $1.001-1.169$ & 0.047 \\
tLNs & & & 0.053 \\
Type of operation & & & 0.096 \\
Age (years) $(>55 v s \leq 55)$ & & & 0.187 \\
Tumor size $(\leqq 4 \mathrm{~cm} v s>4 \mathrm{~cm})$ & & & 0.547 \\
Histological type (undifferentiated $v s$ differentiated) & & & 0.812 \\
Gender & & & 0.810 \\
Tumor location & & & 0.856 \\
\hline
\end{tabular}

HR indicates hazards ratio; 95\% CI, 95\% confidence interval; mLNs: metastatic lymph nodes; tLNs: total retrieved lymph nodes; Variables that were entered into the regression model were EF-Tu, distant metastasis, Serosal invasion, mLNs, tLNs, type of operation, age, tumor size, histological type, Gender, tumor location. Number of mLNs, and number of tLNs were consecutive variables; others were categorized variables.

\section{Discussion}

Elongation factor $\mathrm{Tu}(\mathrm{EF}-\mathrm{Tu})$ is involved in mitochondrial protein synthesis, which complexes with GTP and transports the aa-tRNA to the programmed ribosome [15,16]. Moreover, it can participate in other cellular processes such as organization of mitotic apparatus, developmental regulation, aging, cell morphology and transformation [17-19]. Recent studies have shown that dysregulation of EF-Tu is involved in the oncogenic process [14]. However, both decreased and increased levels of EF-Tu expression have been found in different human cancers, and these contradictory results led to different 
conclusions for the roles of EF-Tu in carcinogenesis. In hepatocellular carcinoma, down-regulation of EF-Tu expression was found in cell line HepG2, while up-regulation was found in another cell line HCC-S102 [12]. In addition, EF-Tu was up-regulated in human gastric cancer cell line AGS compared to the rat normal gastric cell line RGM-1 by two-dimensional electrophoresis proteomic analysis on the mitochondria-enriched fractions, which may influence the function of the mitochondrial [14]. In our preliminary study, we also observed different levels of EF-Tu expression in some tumor cell lines, including high level EF-Tu expression in breast cancer cell line MCF7, leukemia cell line k562 and gastric cancer cell lines SGC-7901, MKN-45 and KatoIII, and low level EF-Tu expression in gastric cancer cell lines MKN-28 and MKN-74. All these investigations indicate that the role EF-Tu plays in the oncogenic process, may be very complicated.

In the current study, we examined EF-Tu expression in 104 gastric adenocarcinoma cases and showed that EF-Tu staining in specimens is predominantly localized on the cell membrane/cytoplasm, and the level of EF-Tu expression decreased in tumor specimens as compared to normal counterparts. Down-regulation of EF-Tu expression in tumor specimens showed a powerful adverse prognostic effect in our study. The effect of EF-Tu expression status was independent of other known predictors, such as the nodal ratio, tumor size, depth of invasion or distant metastasis. Furthermore, EF-Tu expression level was associated with curative resection (R0) patients' outcome. The interesting thing was that all six EF-Tu-negative patients recurred after operation. To increase case numbers in future may provide more clear and solid evidence.

Although there have been several studies on EF-Tu expression in different cancer cell lines [20-22], no investigation on EF-Tu expression in gastric adenocarcinoma specimens and correlation analysis between EF-Tu and adenocarcinoma patients' clinicopathological parameters and prognosis has been carried out to date. Recent molecular-profiling studies have identified EF-Tu dysregulation as a potentially important event in cancer development and proposed it may serve as potential cancer biomarkers in mechanism of the mitochondrial changes in gastric tumor cell [14], but this postulation lacks clinical evidence. It is not yet clear how EF-Tu expression level change affects the survival of patients with gastric adenocarcinoma. Since EF-Tu displays chaperone activity in the quality control of misfolded newly synthesized polypeptides in mitochondria in a GTP-independent manner [23,24], we speculated that when EF-Tu was down-regulation, the peptides synthesized from mitochondria ribosomes may be misfolded, which may lead to tumor development and/or progression [25,26].

Mitochondria are key players in several cellular functions including growth, division, energy metabolism and apoptosis, and its dysfunction contributes to a number of human disorders and may aid cancer progression [27]. It is believed that gastric adenocarcinoma is associated with a severe hypoxia and mitochondria degeneration, when comparing with normal subjects [28,29]. EF-Tu participates in the elongation process of protein biosynthesis in mitochondria, and down-regulation of EF-Tu may suggest the dysfunctions of mitochondria, which might be another reason for poor survival in patients with EF-Tu down-regulation.

One of the main characteristics of cancer cells is their rapid proliferation and this easily causes local hypoxic environment, due to the inability of the local vasculature to supply an adequate amount of oxygen. Because of the inability of mitochondria to provide enough ATP for cell survival under hypoxic conditions, tumor cells must up-regulate the glycolytic pathway [30,31]. This occurs by 
induction of hypoxia-inducible factor 1 (HIF-1) which also suppresses mitochondrial function in tumor cells $[32,33]$, suggesting a hypoxic relationship with mitochondrial dysfunction.

In summary, we have shown that expression of EF-Tu can be used as a potential molecular marker to predict patient outcome in gastric adenocarcinoma patients. Our data showed that negative or low EF-Tu expression has a high correlation with poor survival both in univariate and multivariate analyses. Therefore future investigation on elucidation of the precise roles of EF-Tu expression in gastric adenocarcinoma will provide new insights, which will contribute to improved diagnosis and treatment.

\section{Acknowledgement}

We thank to the Grant Support from Science and Technology Development Program of Hangzhou (20091233Q35).

\section{References}

1. Hohenberger, P.; Gretschel, S. Gastric cancer. Lancet 2003, 362, 305-315.

2. Dicken, B.J.; Bigam, D.L.; Cass, C.; Mackey, J.R.; Joy, A.A.; Hamilton, S.M. Gastric adenocarcinoma: Review and considerations for future directions. Ann. Surg. 2005, 241, 27-39.

3. Zhang, B.; Cao, W.; Zhang, F.; Zhang, L.; Niu, R.; Niu, Y.; Fu, L.; Hao, X.; Cao, X. Protein interacting with $\mathrm{C}$ alpha kinase 1 (PICK1) is involved in promoting tumor growth and correlates with poor prognosis of human breast cancer. Cancer Sci. 2010, 101, 1536-1542.

4. Parmeggiani, A.; Nissen, P. Elongation factor Tu-targeted antibiotics: Four different structures, two mechanisms of action. FEBS Lett. 2006, 580, 4576-4581.

5. Suzuki, H.; Ueda, T.; Taguchi, H.; Takeuchi, N. Chaperone properties of mammalian mitochondrial translation elongation factor Tu. J. Biol. Chem. 2007, 282, 4076-4084.

6. Caldas, T.D.; El Yaagoubi, A.; Richarme, G. Chaperone properties of bacterial elongation factor EF-Tu. J. Biol. Chem. 1998, 273, 11478-11482.

7. Archambaud, C.; Gouin, E.; Pizarro-Cerda, J.; Cossart, P.; Dussurget, O. Translation elongation factor EF-Tu is a target for Stp, a serine-threonine phosphatase involved in virulence of Listeria monocytogenes. Mol. Microbiol. 2005, 56, 383-396.

8. Sakai, J.; Ishikawa, H.; Kojima, S.; Satoh, H.; Yamamoto, S.; Kanaoka, M. Proteomic analysis of rat heart in ischemia and ischemia-reperfusion using fluorescence two-dimensional difference gel electrophoresis. Proteomics 2003, 3, 1318-1324.

9. Shin, J.H.; London, J.; Le Pecheur, M.; Höger, H.; Pollak, D.; Lubec, G. Aberrant neuronal and mitochondrial proteins in hippocampus of transgenic mice overexpressing human $\mathrm{Cu} / \mathrm{Zn}$ superoxide dismutase 1. Free Radic. Biol. Med. 2004, 37, 643-653.

10. Wells, J.; Henkler, F.; Leversha, M.; Koshy, R. A mitochondrial elongation factor-like protein is over-expressed in tumours and differentially expressed in normal tissues. FEBS Lett. 1995, 358, $119-125$.

11. Grant, A.G.; Flomen, R.M.; Tizard, M.L.; Grant, D.A. Differential screening of a human pancreatic adenocarcinoma lambda gt11 expression library has identified increased transcription of elongation factor EF-1 alpha in tumour cells. Int. J. Cancer 1992, 50, 740-745. 
12. Srisomsap, C.; Sawangareetrakul, P.; Subhasitanont, P.; Panichakul, T.; Keeratichamroen, S.; Lirdprapamongkol, K.; Chokchaichamnankit, D.; Sirisinha, S.; Svasti, J. Proteomic analysis of cholangiocarcinoma cell line. Proteomics 2004, 4, 1135-1144.

13. Koch, I.; Hofschneider, P.H.; Lottspeich, F.; Eckerskorn, C.; Koshy, R. Tumour-related expression of a translation-elongation factor-like protein. Oncogene 1990, 5, 839-843.

14. Kim, H.K.; Park, W.S.; Kang, S.H.; Warda, M.; Kim, N.; Ko, J.H.; Prince, Ael-B.; Han, J. Mitochondrial alterations in human gastric carcinoma cell line. Am. J. Physiol. Cell Physiol. 2007, 293, C761-C771.

15. Parmeggiani, A.; Nissen, P. Elongation factor Tu-targeted antibiotics: Four different structures, two mechanisms of action. FEBS Lett. 2006, 580, 4576-4581.

16. Zuurmond, A.M.; Martien de Graaf, J.; Olsthoorn-Tieleman, L.N.; van Duyl, B.Y.; Mörhle, V.G.; Jurnak, F.; Mesters, J.R.; Hilgenfeld, R.; Kraal, B. GE2270A-resistant mutations in elongation factor Tu allow productive aminoacyl-tRNA binding to EF-Tu.GTP.GE2270A complexes. J. Mol. Biol. 2000, 304, 995-1005.

17. Zipfel, C.; Kunze, G.; Chinchilla, D.; Caniard, A.; Jones, J.D.; Boller, T.; Felix, G. Perception of the bacterial PAMP EF-Tu by the receptor EFR restricts Agrobacterium-mediated transformation. Cell 2006, 125, 749-760.

18. Wells, J.; Henkler, F.; Leversha, M.; Koshy, R. A mitochondrial elongation factor-like protein is over-expressed in tumours and differentially expressed in normal tissues. FEBS Lett. 1995, 358, $119-125$.

19. Gross, S.R.; Kinzy, T.G. Translation elongation factor $1 \mathrm{~A}$ is essential for regulation of the actin cytoskeleton and cell morphology. Nat. Struct. Mol. Biol. 2005, 12, 772-778.

20. Kuramitsu, Y.; Baron, B.; Yoshino, S.; Zhang, X.; Tanaka, T.; Yashiro, M.; Hirakawa, K.; Oka, M.; Nakamura, K. Proteomic differential display analysis shows up-regulation of 14-3-3 sigma protein in human scirrhous-type gastric carcinoma cells. Anticancer Res. 2010, 30, 4459-4465.

21. Rao, T.R.; Slobin, L.I. The stability of mRNA for eucaryotic elongation factor Tu in Friend erythroleukemia cells varies with growth rate. Mol. Cell. Biol. 1988, 8, 1085-1092.

22. Omori, S.; Sato, Y.; Hirakawa, S.; Isobe, T.; Yukawa, M.; Murata, K. Two extra chromosomal genomes of Leucocytozoon caulleryi; complete nucleotide sequences of the mitochondrial genome and existence of the apicoplast genome. Parasitol. Res. 2008, 103, 953-957.

23. Krab, I.M.; Te Biesebeke, R.; Bernardi, A.; Parmeggiani, A. Elongation factor Ts can act as a steric chaperone by increasing the solubility of nucleotide binding-impaired elongation factor-Tu. Biochemistry 2001, 40, 8531-8535.

24. Krab, I.M.; Te Biesebeke, R.; Bernardi, A.; Parmeggiani, A. Chloroplast protein synthesis elongation factor, EF-Tu, reduces thermal aggregation of rubisco activase. J. Plant Physiol. 2007, 164, 1564-1571.

25. Modica-Napolitano, J.S.; Kulawiec, M.; Singh, K.K. Mitochondria and human cancer. Curr. Mol. Med. 2007, 7, 121-131.

26. Modica-Napolitano, J.S.; Singh, K.K. Mitochondrial dysfunction in cancer. Mitochondrion 2004, 4, 755-762. 
27. Gogvadze, V.; Orrenius, S.; Zhivotovsky, B. Mitochondria in cancer cells: What is so special about them? Trends Cell Biol. 2008, 18, 165-173.

28. Calabrese, C.; Pisi, A.; Di Febo, G.; Liguori, G.; Filippini, G.; Cervellera, M.; Righi, V.; Lucchi, P.; Mucci, A.; Schenetti, L.; et al. Biochemical alterations from normal mucosa to gastric cancer by ex vivo magnetic resonance spectroscopy. Cancer Epidemiol. Biomarkers Prev. 2008, 17, 1386-1395.

29. Zhang, L.; Li, L.; Liu, H.; Prabhakaran, K.; Zhang, X.; Borowitz, J.L.; Isom, G.E. HIF-1alpha activation by a redox-sensitive pathway mediates cyanide-induced BNIP3 upregulation and mitochondrial-dependent cell death. Free Radic. Biol. Med. 2007, 43, 117-127.

30. Gillies, R.J.; Robey, I.; Gatenby, R.A. Causes and consequences of increased glucose metabolism of cancers. J. Nucl. Med. 2008, 49, 24S-42S.

31. Kondoh, H. Cellular life span and the Warburg effect. Exp. Cell Res. 2008, 314, 1923-1928.

32. Kim, J.W.; Tchernyshyov, I.; Semenza, G.L.; Dang, C.V. HIF-1-mediated expression of pyruvate dehydrogenase kinase: A metabolic switch required for cellular adaptation to hypoxia. Cell Metab. 2006, 3, 177-185.

33. Papandreou, I.; Cairns, R.A.; Fontana, L.; Lim, A.L.; Denko, N.C. HIF-1 mediates adaptation to hypoxia by actively down regulating mitochondrial oxygen consumption. Cell Metab. 2006, 3, 187-197.

(C) 2011 by the authors; licensee MDPI, Basel, Switzerland. This article is an open access article distributed under the terms and conditions of the Creative Commons Attribution license (http://creativecommons.org/licenses/by/3.0/). 